\title{
Probabilistic Properties of Highly Connected Random Geometric Graphs
}

\author{
Bodo Manthey $^{1}$ and Victor M.J.J. Reijnders ${ }^{1}$ \\ ${ }^{1}$ University of Twente, Department of Applied Mathematics, Enschede, The Netherlands
}

\begin{abstract}
In this paper we study the probabilistic properties of reliable networks of minimal total edge lengths. We study reliability in terms of $k$-edge-connectivity in graphs in $d$-dimensional space. We show this problem fits into Yukich's framework for Euclidean functionals for arbitrary $k$, dimension $d$ and distant-power gradient $p$, with $p<d$. With this framework several theorems on the convergence of optimal solutions follow. We apply Yukich's framework for functionals so that we can use partitioning algorithms that rapidly compute near-optimal solutions on typical examples. These results are then extended to optimal $k$-edge-connected power assignment graphs, where we assign power to vertices and charge per vertex. The network can be modelled as a wireless network.
\end{abstract}

\section{Introduction}

The design of fault tolerant networks is an important issue in today's research, due to their numerous applications [1]. The goal is to find cheap and reliable networks with some specific characteristics. Reliability is often expressed in terms of the connectivity of a network. For example, we might want to have multiple paths between each pair of nodes to account for possible failures in a link.

Wireless ad hoc networks have also received significant attention in recent studies [4, 6]. Instead of direct connections between nodes, communication takes place through single-hop transmissions or by relaying through intermediate nodes. Here we assign a transmission power to each node. As transmission range is directly related to power usage and therefore to battery lifetime, the goal is to find a fault tolerant network with minimal total power usage.

Finding a cheapest $k$-edge-connected network is NP-hard [5], and so is finding a minimal power wireless network [3]. As we still want to have reasonably good solutions in acceptable computation time, we need to find good heuristics. We fit the problems into Yukich's framework for Euclidean functional [8] to get limit theorems and concentration results, as well as using them for analysis of the partitioning algorithm.

Partitioning algorithms have shown a lot of potential with similar problems [2]. In practice, partitioning algorithms are very fast. Partitioning algorithms divide the whole problems into smaller cells and compute optimal solutions on these. Then these solutions are joined to obtain a solution for the whole problem. 


\section{Definitions and Results}

All graphs in this paper are undirected and simple. Let $G=(V, E)$ be a graph. We assume $V \subset \mathbb{R}^{d}$, where $d$ is a constant and $V$ is finite. The cost of an edge is its length raised to the power of the distant-power gradient $p>0$. So adding edge $(u, v)$ to a graphs increases the cost by $|(u, v)|^{p}$, where $|(u, v)|$ denotes the Euclidean distance between $u$ and $v$. Here, we assume $p$ is a constant.

A graph is $k$-edge-connected if the graph is still connected when at most $k-1$ edges are removed, or if it is complete. The latter is to make sure $k$-edge-connected graphs on less than $k+1$ nodes still exist, which saves us from dealing with all kind of exceptions in proofs. Alternatively, a network is $k$-edge-connected if there exist at least $k$ edge-disjoint paths between every pair of vertices.

Let $d \in \mathbb{N}$ be arbitrary and let $p>0$. Then $\operatorname{MkEE}^{p}(V)$ is the minimal length of a $k$ edge-connected graph in terms of summed edge lengths on $V$ with $p$ th power-weighted edges. Thus

$$
\operatorname{MkEE}^{p}(V)=\min _{X \in \mathcal{S}(V, k)} \sum_{e \in X}|e|^{p}
$$

where $\mathcal{S}(V, k)$ is the set of $k$-edge-connected simple graphs on $V$ and $|e|$ denotes the Euclidean length of an edge $e$. Following Yukich [8], we call $\mathrm{MkEE}^{p}$ a functional.

One of the desired properties for functionals is subadditivity. Roughly speaking, this shows that the function value of a whole set is not larger than the sum of function values of the sets in a partition of this set (with some error term).

Theorem 1. For $p \geq 1, \mathrm{MkEE}^{p}$ is geometrically subadditive, i.e. for all finite sets $V$, all rectangles $R$ and all partitions of $R$ into rectangles $R_{1}$ and $R_{2}$ we have

$$
\operatorname{MkEE}^{p}(V \cap R) \leq \operatorname{MkEE}^{p}\left(V \cap R_{1}\right)+\operatorname{MkEE}^{p}\left(V \cap R_{2}\right)+C_{1}(\operatorname{diam} R)^{p},
$$

where $C_{1}=C_{1}(d, p)$ is a constant.

We would also want $\mathrm{MkEE}^{p}$ to be superadditive. Roughly speaking, this would show that the function value of a whole set is not lower than the sum of function values of the sets in a partition. Combining sub- and superadditivity makes the functional nearly additive in the sense that $\operatorname{MkEE}^{p}(F, R) \approx \operatorname{MkEE}^{p}\left(F, R_{1}\right)+\operatorname{MkEE}^{p}\left(F, R_{2}\right)$.

We could then approximate the optimal solution value of the whole set by the sum of optimal solutions on its partitions. It is easily checked however that $\mathrm{MkEE}^{p}$ does not possess superadditivity. This is why we introduce the canonical boundary functional, an idea first articulated in Redmond's thesis [7]. In boundary functionals, the entire boundary of the rectangle is considered as one additional vertex that can be used. We also refer to Yukich [8] for more on this topic.

$\mathrm{MkEE}_{B}^{p}$ is the boundary functional of $\mathrm{MkEE}^{p}$, so that $\operatorname{MkEE}_{B}^{p}(V \cap R)$ is the minimal length of a $k$-edge-connected boundary graph in terms of summed edge lengths on $V \cup \partial R$ in $d$ dimensional rectangle $R$ with $p$ th power-weighted edges. Here $\partial R$ denotes the boundary of $R$. A vertex $v$ is connected to $\partial R$ by adding edge $\left(v, v_{\partial}\right)$ where $v_{\partial}=\arg \min _{w \in \partial R}|(v, w)|$.

Theorem 2. For $p \geq 1, \mathrm{MkEE}_{B}^{p}$ is a superadditive functional, i.e. for all finite sets $V$, all rectangles $R$ and all partitions of $R$ into rectangles $R_{1}$ and $R_{2}$ we have

$$
\operatorname{MkEE}_{B}^{p}(V \cap R) \geq \operatorname{MkEE}_{B}^{p}\left(V \cap R_{1}\right)+\operatorname{MkEE}_{B}^{p}\left(V \cap R_{2}\right) .
$$


As we cannot directly show near additivity, we want to show that $\mathrm{MkEE}^{p}$ and $\mathrm{MkEE}_{B}^{p}$ are pointwise close. Then we would get approximately get sub- and superadditivity for both functionals.

Theorem 3. For $1 \leq p<d, \mathrm{MkEE}^{p}$ is pointwise close to $\mathrm{MkEE}_{B}^{p}$, i.e. for all finite sets $V \subset[0,1]^{d}$ we have

$$
\left|\operatorname{MkEE}^{p}(V)-\operatorname{MkEE}_{B}^{p}(V)\right|=o\left(|V|^{(d-p) / d}\right) .
$$

We have shown geometric subadditivity, superadditivity and pointwise closeness, creating a powerful set of properties. These properties are more useful for obtaining other results when the functional also is smooth. This describes how strong the variations of a functional are if vertices are added or deleted. Smooth functionals behave a lot more predictable and therefore it plays an important role in many limit theories.

Theorem 4. For $1 \leq p<d, \mathrm{MkEE}^{p}$ is smooth, i.e. for all finite sets $U$ and $V$ we have

$$
\left|\operatorname{MkEE}^{p}(U \cup V)-\operatorname{MkEE}^{p}(U)\right|=O\left(|V|^{(d-p) / d}\right) .
$$

One of the concentration results we have obtained is stated below. It shows that the functional values are not far from their expected value.

Theorem 5. For $1 \leq p<d$ and $k \in \mathbb{N}$, there exists a constant $\alpha=\alpha(d, k) \geq 0$ such that

$$
\begin{aligned}
& \lim _{n \rightarrow \infty} \operatorname{MkEE}^{p}(V, R) / n^{(d-p) / p}=\alpha \quad \text { c.c., and } \\
& \lim _{n \rightarrow \infty} \operatorname{MkEE}_{B}^{p}(V, R) / n^{(d-p) / p}=\alpha \quad \text { c.c., }
\end{aligned}
$$

where $n=|V|$. Here c.c. denotes complete convergence.

\section{Partitioning algorithm}

In a partitioning algorithm, the Euclidean plane is divided into a number of cells that all contain only a few points. On each cell an optimal solution is calculated. This is generally much faster than calculating a solution on all points at once, as these problems are often NP-hard. The solutions of all cells are then joined to obtain a solution for the whole set.

We implement a partitioning scheme for $\mathrm{MkEE}^{p}$ having a polynomial running time, for which we derive approximation guarantees.

Algorithm 6 (Partitioning Scheme).

Input: set $V \subseteq[0,1]^{d}$ of $n$ points and number of points per cell $s$

1. Partition $[0,1]^{d}$ into $\ell=\sqrt[d]{n / s}$ stripes of dimension $d-1$ such that each stripe contains exactly $n / \ell=\left(n^{d-1} s\right)^{1 / d}$ points.

2. Keep partitioning each $i+1$-dimensional stripe into $\ell$ stripes of dimension $i$ such that each stripe contains exactly $n / \ell^{i}=\left(n^{d-i} s^{i}\right)^{1 / d}$ points. Stop at $i=1$ so that each 2 dimensional stripe is partitioned into $\ell$ cells with $n / \ell^{d}=s$ points. In this way we end up with $\ell^{d}=n / s$ cells. Here we assume $s>k$.

3. Compute a graph achieving the optimal solution of $\mathrm{MkEE}^{p}$ for each cell.

4. Join the graphs to obtain a k-edge-connected graph on $V$. 
It can be easily verified that the graph we get as an output from Algorithm 6 is $k$-edgeconnected. With this algorithm and the properties obtained in Section 2 we can now give running time and approximation guarantees. Depending on the way we compute the optimal solution on each cell, we need to vary $s$ to get a polynomial running-time.

Theorem 7. If the algorithm for computing an optimal solution on each cell in Algorithm 6 has a running time of $O\left(C^{n^{2}}\right)$ for some constant $C$, the Partitioning Scheme has a polynomial running time if we choose $s=O(\sqrt{\log n})$. The approximation guarantee then becomes $\operatorname{MkEE}^{p}(V)+O\left((n / s)^{(d-p) / d}\right)$ for $k$-edge-connected graphs.

\section{Extention to wireless networks}

Besides our model for wired networks, we consider a different model for wireless networks. These are defined by assigning power to each vertex. A power assignment PA assigns a real, positive value to all vertices $v \in V$. The corresponding power assignment graph then contains all edges $(u, v)$ for which $\mathrm{PA}(u), \operatorname{PA}(v) \geq|(u, v)|^{p}$. The costs of $k$-edge-connected power assignment graphs is then simply the sum of all assigned powers. We obtain results similar to Theorems $1-5$ for the functional in wireless networks.

\section{References}

[1] Fatiha Bendali, I Diarrassouba, Ali Ridha Mahjoub, M Didi Biha, and Jean Mailfert, A branch-and-cut algorithm for the k-edge connected subgraph problem, Networks 55 (2010), no. $1,13-32$.

[2] Markus Bläser, Bodo Manthey, and BV Raghavendra Rao, Smoothed analysis of partitioning algorithms for euclidean functionals, Algorithmica 66 (2013), no. 2, 397-418.

[3] Andrea EF Clementi, Paolo Penna, and Riccardo Silvestri, On the power assignment problem in radio networks, Mobile Networks and Applications 9 (2004), no. 2, 125-140.

[4] Maurits de Graaf and Bodo Manthey, Probabilistic analysis of power assignments, International Symposium on Mathematical Foundations of Computer Science, Springer, 2014, pp. 201-212.

[5] Michael R Gary and David S Johnson, Computers and intractability: A guide to the theory of np-completeness, 1979.

[6] Ram Ramanathan and Regina Rosales-Hain, Topology control of multihop wireless networks using transmit power adjustment, INFOCOM 2000. Nineteenth Annual Joint Conference of the IEEE Computer and Communications Societies. Proceedings. IEEE, vol. 2, IEEE, 2000, pp. 404-413.

[7] Charles Redmond, Boundary rooted graphs and euclidean matching algorithms, Ph.D. thesis, Lehigh University, Bethlehem, PA, USA, 1993.

[8] Joseph E Yukich, Probability theory of classical euclidean optimization problems, Lecture Notes in Mathematics, vol. 1675, Springer-Verlag, Berlin, 1998. 